\title{
A New Face Recognition Algorithm Based on Haar-Like Features and SRC with Gentle Adaboost
}

\author{
G.M. Zhu \\ School of Information Engineering \\ Wuyi University \\ Jiangmen, China
}

\author{
Z.L. Ying \\ School of Information Engineering \\ Wuyi University \\ Jiangmen, China
}

\author{
L.W. Huang \\ School of Information Engineering \\ Wuyi University \\ Jiangmen, China
}

\begin{abstract}
In this paper, a new face recognition algorithm based on Haar-Like features and Gentle Adaboost (GA) feature selection via sparse representation was proposed. Firstly, all the images including face images and non-face images were normalized to size $20 \times 20$, then Haar-Like features were extracted from the images after size normalization. The number of the extracted features is more than 125,199. Then, Gentle Adaboost algorithm was exploited to feature selection that reduced the feature dimension and retained the most effective features for face recognition. Finally, the samples were classified and identified for face recognition via sparse representation classification (SRC) algorithm. Comparing it with SRC, Nearest Neighbor (NN), Nearest Subspace (NS), Support Vector Machine (SVM) algorithms, the experiment results on AR database demonstrate that the new proposed algorithm can achieve a higher recognition rate than these traditional algorithms. Even though the increase of the dimension, the new proposed algorithm always got higher recognition rate than SRC and other algorithms, which proved the new algorithm can get better identification effects and stronger stability in the low dimension feature space.
\end{abstract}

Keywords-Haar-Like features; Gentle Adaboost; sparse representation; face recognition

\section{INTRODUCTION}

In this paper, all the Haar-Like features are used which proposed so far in face recognition in reference[1,2]. Gentle Adaboost algorithm for feature selection is also used. The rests of the paper are organized as follows. In section 2, Haar-Like features are described. In section 3 and 4, the fundamental theories of Gentle Adaboost classifier and Sparse Representation Classification algorithm are described for face recognition and classification. Some extensive experiment analysis and results are given in section 5 and 6 . Finally, some conclusions are summarized in section 7.

\section{HAAR-LIKE FEATURES}

Haar-Like features in document[3,4] are common feature descriptors in the fields of computer vision. Haar-Like features on the "block" features can reduce the computational costs effectively. The Haar-Like features are equal to the subtraction of the summation of black pixels gray values and the summation of white pixels gray values. Haar-Like features are mainly divided into four categories, i.e., edge features (as shown in Fig 1. (a) to (d)), line features(Fig1. (e) to (l)), point features (Fig1. (m) to (n)) and diagonal feature(Fig1. (o)).

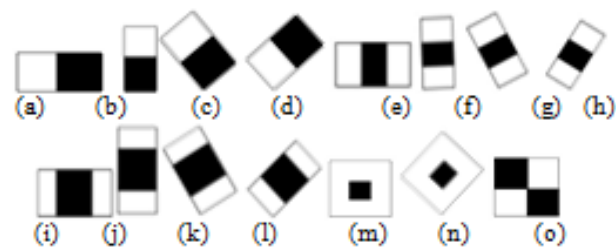

\section{FIGURE I. ALL THE HAAR-LIKE FEATURES PROPOSED NOWADAYS}

\section{GENTLE ADABOOST}

The feature dimension of Haar-Like features is quite high. For an image of size $20 \times 20$, the number of features is equal to 125,199. In order to choose the effective features for face recognition from the plenty of features, Gentle Adaboost algorithm is adopted for feature selection. Gentle Adaboost is also called smooth Adaboost in reference[5], the algorithm that is the phase estimation process of an additive logistic regression model verifies the entire process of Adaboost algorithm in a statistical point of view. By the analysis and verification of Discrete Adaboost algorithm, Gentle Adaboost algorithm is thus introduced.

In the Gentle Adaboost algorithm, the definition of weak classifiers is shown as follows:

$$
h\left(x_{i}\right)=\left\{\begin{array}{l}
\alpha_{1}, f\left(x_{i}\right)<\theta \\
\alpha_{2}, f\left(x_{i}\right) \geq \theta
\end{array}\right.
$$




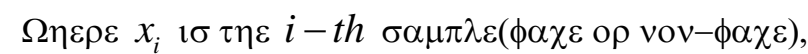

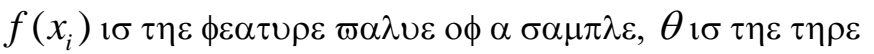

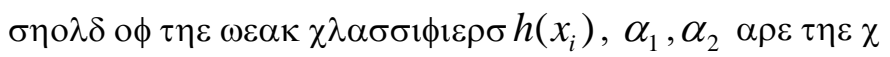

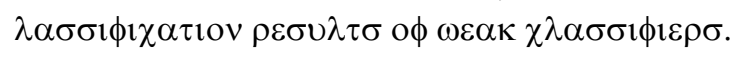

\section{SPARSE REPRESENTATION AlgORITHM}

The general steps of sparse representation classification algorithm are shown as follows:

1). Input the matrix of the given training samples $A=\left[A_{1}, A_{2}, \ldots, A_{k}\right]$, where $A_{i} \in R^{m \times n \times k}$, which contains $k$ classes, and give a test sample $y \in R^{m \times n}$.

2). Make each column of the matrix $A$ and $y$ to have

$l_{2}$-norm normalization .

3). Solve the $l_{1}$-norm minimization problem:

$\hat{x}=\arg \min \|x\|_{1}$ subject to $A x=y, x$ is the solution of a linear equation.

4). Compute the residuals:

$$
r_{i}=\left\|y-A \delta_{i}(x)\right\|_{2}, i=1, \ldots k
$$

where $\delta_{i}(x) \in R$ in formula (2) is a new vector, which the nonzero item is associated with vector $X$ and the class $i$ item.

5). Output the identity:

$$
\text { identity }(y)=\arg \min _{i} r_{i}(y) .
$$

In the end, all Haar-Like features are extracted through the Gentle Adaboost algorithm for the feature selection, then these selected features are classified and recognized in formula (3) via sparse representation algorithm.

\section{EXPERIMENTS}

We normalize the images into $20 \times 20$ on the AR database, and take all images on the AR database as positive samples, the total number is 2600; 4000 images are collected from the internet which have same size $20 \times 20$ as negative samples that cannot contain the face of non-face images. In order to train 465(it is the self-adaptive process) weak classifiers, we set up 465 as the number of training rounds.

We select 100 individuals(50 men and 50 women) on AR database and use 7 images randomly selected of each person taken in one period including facial expression changes and illumination as training samples. We randomly select 7 images of each person who comes from the other period including facial expression changes, illumination as a test. In this paper, we use the same test sets and the same training sets in comparing with the gray features combining SRC algorithm.

\section{ANALYSIS AND RESULTS}

\section{A. The Statistical Table of Face Recognition Rate}

The experiment results are shown in Table 1 . We can see from the experiment results that the face recognition rate of the new proposed algorithm is the highest. It is up to $98.14 \%$, which is better than any other face recognition algorithms, such as SRC, NN, NS, SVM[6].

TABLE I. FACE RECOgNition RATE of VARIOUS FACE RECOGNITION ALGORITHMS

\begin{tabular}{|l|l|}
\hline \multicolumn{1}{|c|}{$\begin{array}{c}\text { Face recognition } \\
\text { algorithms }\end{array}$} & \multicolumn{1}{|c|}{$\begin{array}{c}\text { Recognition } \\
\text { rate(\%) }\end{array}$} \\
\hline Haar-Like+GA+SRC & $98.14 \%$ \\
\hline GRAY+SRC & $96 \%$ \\
\hline GRAY+NN [6] & $89.7 \%$ \\
\hline GRAY+NS [6] & $90.3 \%$ \\
\hline GRAY+SVM [6] & $95.7 \%$ \\
\hline
\end{tabular}

\section{B. The Curve Graphs of Face Recognition Rate and Dimension}

The new proposed algorithm also obtains good results in the testing experiments. Figure 2 shows that the effect of face recognition rate changing with feature dimension. To be contrast, face recognition rate curve of SRC algorithm based on gray values is also shown in Figure 2. As you can see from Figure 2, this algorithm corresponding curve always locates on the top. When the feature dimension is low, face recognition rate goes up while feature dimension is increasing. But when it reaches 50, face recognition rate do not change too much with the increasing of feature dimension for the new proposed algorithm. But when the feature dimension is 450 , the recognition rate is up to $99 \%$. It is indicated that the recognition algorithm in this paper can obtain much better recognition rate and much strong stability in low dimension feature space.

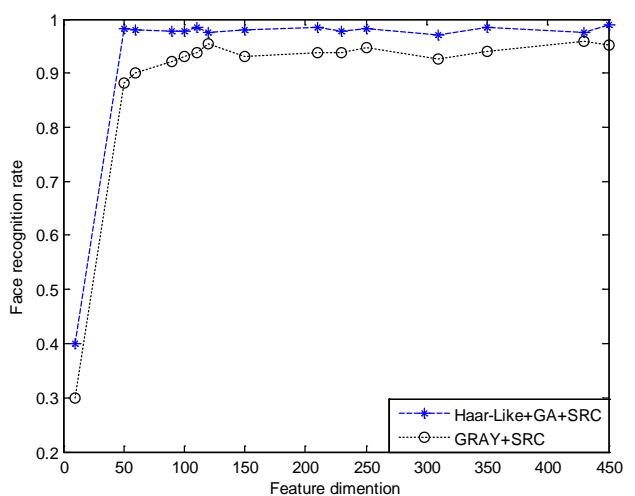

FIGURE II.

FACE RECOGNITION RATE AND THE DIMENSION CURVE GRAPHS

\section{Face Recognition Comparison of Images with Occlusion}

The experiment is to verify the performance of the new proposed algorithm with other face recognition algorithms for damaged images. The testing samples are occlusion to 6 
different degrees, i.e. $0 \%, 10 \%, 20 \%, 30 \%, 40 \%, 50 \%$ respectively. A sample with different degrees of damages is shown in Figure 3.

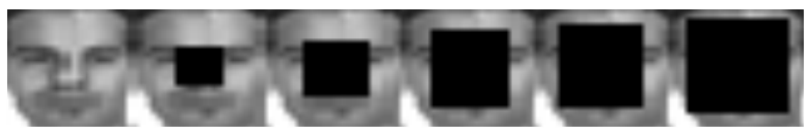

FIGURE III. A SAMPLE FACE IMAGE OF THE AR DATABASE WITH DIFFERENT DEGREES OF OCCLUSION,RESPECTIVELY 0\%, $10 \%, 20 \%, 30 \%, 40 \%, 50 \%$.

The face recognition rates of different algorithms with different degrees of occlusion to the images are shown in Figure 4.

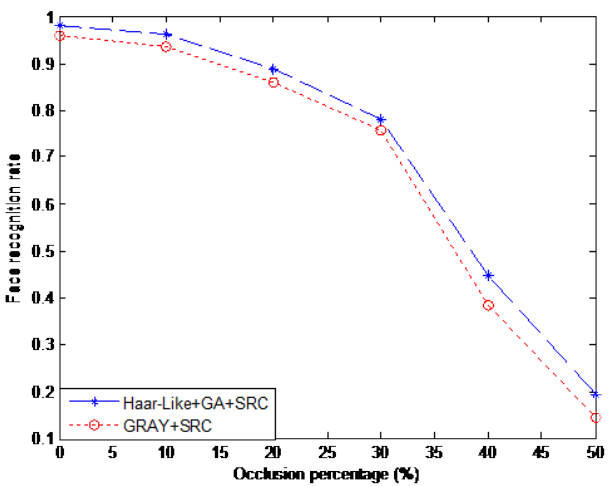

FIGURE IV. THE FACE RECOGNITION RATES OF DIFFERENT ALGORITHMS WITH DIFFERENT DEGREES OF OCCLUSION TO FACE IMAGES

As can be seen from Figure 4, Haar-Like+GA+SRC algorithm gets higher performance compared with GRAY+SRC algorithm. When testing images corrupted by $0 \%, 10 \%, 20 \%$, $30 \%, 40 \%, 50 \%$ respectively, recognition rates of Haar-Like+GA+SRC algorithm are 98.14\%, 96.25\%, 88.7\%, $78.01 \%, 44.6 \%, 19.4 \%$, and recognition rates of GRAY+SRC algorithm are $96 \%$, 93.52\%, 85.9\%, 75.6\%, 38.2\%, 14.3\%. But when the testing images are damaged more than $30 \%$, the recognition rate of GRAY+SRC algorithm is dropping rapidly. So the new proposed algorithm is also the best for occlusion of face recognition.

\section{CONCLUSIONS}

In this paper, we propose a new face recognition algorithm based on Haar-Like features and Gentle Adaboost feature selection. The selected features are put into SRC classifier for face recognition and classification. Testing experiments show that the new proposed algorithm achieves a better performance which compared with the traditional face recognition algorithms, such as SRC, NN, NS, SVM. The effect of face recognition rate changing with feature dimension shows that our algorithm with the increasing of feature dimension performs much better than SRC algorithm all the time, which proves the effectiveness and superiority of the new proposed algorithm.

\section{ACKNOWLEDGMENTS}

This work was supported by Natural Science Fund Project of Guangdong Province, China(NO.S2011010001085, NO.10152902001000002), National Natural Science Fund Project(NO.61072127, NO.61372193), and Guangdong College students' innovative training project.

\section{REFERENCES}

[1] J. Yan, W.S. Cheng, X. Sun: Research and development status of face recognition technology. TV technology, Vol.12(2006), pp. 81-84

[2] W.J. Li, C.J. Wang, W. Zhang, S.F. Chen: A Survey of Face Recognition. Pattern recognition and artificial intelligence, Vol.19(2006), pp. 59-65

[3] Kicheon Hong, Jihong Min, Wonchan Lee, Jungchul Kim: Real Time Face Detection and Recognition System Using Haar-Like Feature/HMM in Ubiquitous Network Environments. Lecture Notes in Computer Science, Vol.3480(2005), pp. 1154-1161

[4] S.R. Zhou, J.P. Yin: LBP Texture Features based on Haar Characteristics. Journal of Software, Vol.24(2013), pp. 1909-1926, .

[5] C.H. Cai, J.Q. Zhu: Real-time face detection using Gentle Adaboost and nested cascade structure Journal of Signal processing, Vol.29(2013), pp. 956-963

[6] J. Wright, A.Y. Yang, A. Geanesh, S.S. Sastry, Yi Ma: Robust Face Recognition via Sparse Representation. IEEE Transactions on Pattern Analysis and Machine Intelligence, Vol.31(2009), pp. 210-227. 